\title{
Session 3: Cancer II
}

Monday 29th October 2007. Moderators: Peter Vollmers and Kathy Potter

[14.00-14.30]

'Title to be confirmed'

Zdenka K. Jonak

GSK, King-of-Prussia, USA

[14.30-14.50]

\author{
Antibody-maytansinoid conjugates as anticancer \\ therapeutics: Proving their benefit \\ John M. Lambert \\ ImmunoGen, Inc., Cambridge, MA, USA
}

Introduction: The idea of using monoclonal antibodies to deliver cytotoxic agents specifically to cancer cells dates to the early 1980s. As with many simple ideas, its successful execution has proved challenging, and a number of approaches have failed to achieve effective, well tolerated anticancer products. Early clinical development in the field of targeted delivery of cytotoxic drugs to tumors using antibodies was not successful because the limitations imposed by the pharmacokinetic and pharmacodynamic properties of monoclonal antibodies were not fully appreciated. In recent years, there has been increasing interest in the use of highly cytotoxic small molecules as the attached cell-killing agent. Several companies now are advancing compounds that comprise a tumor-targeting antibody and derivatives of the potent anti-mitotic microtubule agent, maytansine. One reason for the heightened interest in the field is the increase in the number of companies pursuing antibody-based anticancer agents, since many tumor-targeting antibodies identified as a result of this effort lack meaningful anticancer activity of their own. Creating antibody-maytansinoid conjugate (AMC) compounds provides a means of achieving effective products from such antibodies.

Considerations in the Design of Effective AntibodyMaytansinoid Conjugates: AMC compounds possess an unusually complex mechanism of action: they cir- culate in plasma for long periods, get retained in cancer tissue through binding to tumor-associated cell surface antigen, become internalized as antigen-AMC complexes by the cancer cell, undergo release of the active maytansinoids from the antibody and, finally, have the maytansinoids reach their intracellular target (tubulin) in the cytoplasm of the cell. Therefore, to generate effective anticancer agents, each component of a conjugate - the monoclonal antibody, the cytotoxic effector moiety, the linker, and the conjugate itself (e.g., the number of maytansinoids linked per antibody) have to be optimized.

The Maytansinoid Payload: Maytansinoids are tubulin-binding agents that are potent antimitotics, causing cells to arrest in the G2/M phase of the cell cycle, leading to cell death [1]. They can kill a wide range of tumor cells with $\mathrm{IC}_{50}$ values in the range of 10 $100 \mathrm{pM}$. To link maytansinoids to antibodies, derivatives of maytansine were synthesized that contained a thiol group that provides ready attachment via thioldisulfide chemistry resulting in conjugates containing disulfide linkers. Alternatively, the thiol of the maytansine derivative can react with alkylating moieties of crosslinking agent such as SMCC, resulting in conjugates containing stable thioether linkers. Two different thiol-containing maytansinoids, DM1 and DM4 [Fig. 1] are currently being utilized in AMCs that are undergoing clinical testing.

Antibody-Maytansinoid Conjugates (AMCs): When maytansinoid drugs are linked to antibodies, they acquire selective target-cell directed killing in vitro. Nontarget cells (i.e., cells that do not express the target antigen) are not killed in vitro, even when sensitive to maytansine itself, except at very high concentrations of immunoconjugate where non-antigen-dependent cellular uptake mechanisms can occur. Thus, AMCs behave as tumor-activated prodrugs. In vivo, distribution studies in tumor xenograft models in SCID mice show that linking maytansinoids to antibodies also profoundly enhances the amount of the cytotoxin that can accu- 


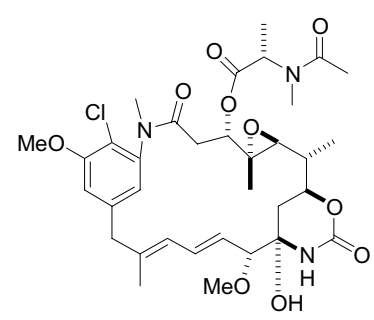

Maytansine

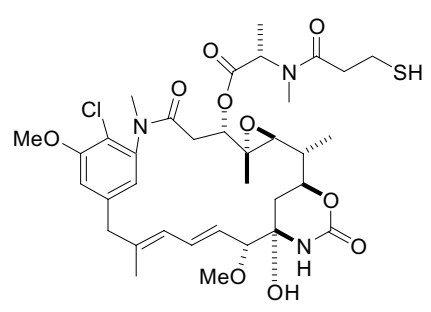

DM1

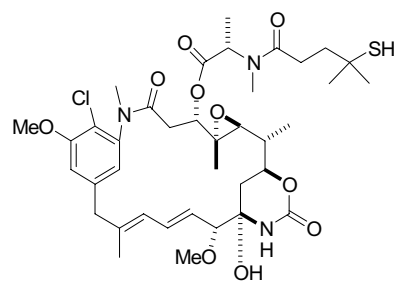

DM4

Fig. 1. Chemical Structures of Maytansine, DM1, and DM4.

mulate at a tumor when compared with non-conjugated maytansinoids - about a 1000-fold greater accumulation of maytansinoid can be achieved at tumor after 24$28 \mathrm{~h}$. Thus, linking maytansinoids to antibodies alters their in vivo distribution, essentially conferring the distribution properties of an antibody to the maytansinoid drug.

The altered distribution of maytansinoids upon conjugation to an antibody results in a larger therapeutic window for the AMC compared to that of the free drug. The enhanced localization of maytansinoids at tumors via conjugation explains the extraordinary potent antitumor activity of these conjugates [2,3]. Figure 2 shows an example of such activity in mice bearing a colon tumor xenograft. Treatment of the mice with a single dose of the immunoconjugate huC242-DM4 (12 mg/kg conjugated antibody) resulted in marked, complete regression of the tumor at a dose well below the maximum tolerated dose (about 50-60 mg/kg), while equivalent doses of non-conjugated DM4, the "naked" huC242 antibody, and a DM4 conjugate with a non-binding antibody all had no effect on tumor growth in this model. In preclinical evaluation, AMC compounds consistently demonstrate anticancer activity far in excess of that achieved by either the antibody component or the maytansinoid component alone, while exhibiting an excellent safety profile.

SCID mice bearing established subcutaneous COLO 205 xenografts treated with huC242-DM4 as a single

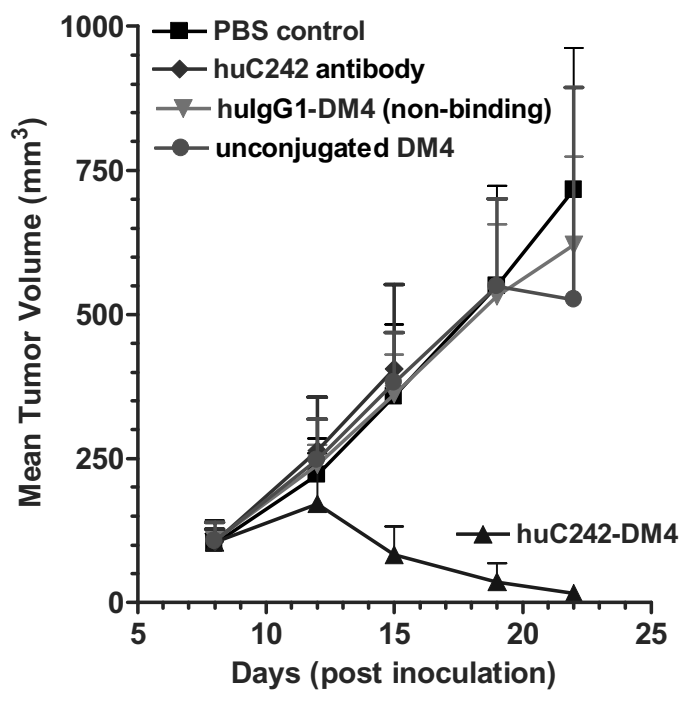

Fig. 2. Potent Antitumor Activity of Conjugate between the huC242 Antibody and the Maytansinoid DM4.

bolus IV injection (12 mg/kg MAb/0.2 mg/kg DM4 dose), or equivalent doses of control compounds.

The Linker Moiety: When considering the design of the linker between the antibody and the maytansinoid, one must take into accounts three aspects: the sites of the attachment of the linker to the antibody, the number of cytotoxic molecules linked per antibody molecules, 
Table 1

Antibody-Drug Conjugates Undergoing Clinical Development

\begin{tabular}{|c|c|c|c|c|c|}
\hline $\begin{array}{l}\text { Drug-Antibody } \\
\text { Conjugate }\end{array}$ & $\begin{array}{l}\text { Drug } \\
\text { Type }\end{array}$ & Antibody/Target & Cancer & Developer & Development Status \\
\hline BB-10901 [huN901-DM1] & $\mathrm{DM}^{(\mathrm{c})}$ & huN901/CD56 & $\begin{array}{l}\operatorname{SCLC}^{(\mathrm{b})} \text {, } \\
\text { neuroendocrine multiple } \\
\text { myeloma }\end{array}$ & $\operatorname{ImmunoGen}^{(a)}$ & $\begin{array}{l}\mathrm{Ph} \mathrm{I} \& \mathrm{Ph} \text { II trials in } \\
\text { progress }\end{array}$ \\
\hline $\begin{array}{l}\text { AVE9633 } \\
\text { [huMy9-6-DM4] }\end{array}$ & $\mathrm{DM} 4^{(\mathrm{d})}$ & huMy9-6/CD33 & $\mathrm{AML}^{(\mathrm{b})}$ & sanofi-aventis & $\mathrm{Ph} \mathrm{I}$ \\
\hline huC242-DM4 & DM4 & huC242/CanAg & $\begin{array}{l}\text { Colorectal, pancreatic, } \\
\text { other CanAg }{ }^{+} \text {tumors }\end{array}$ & ImmunoGen & $\begin{array}{l}\mathrm{Ph} \mathrm{I} \& \mathrm{Ph} \mathrm{II} \mathrm{(gastric)} \mathrm{trials} \\
\text { in progress }\end{array}$ \\
\hline Tmab-DM1 & DM1 & Trastuzumab/HER2 & Breast & Genentech & $\begin{array}{l}\mathrm{Ph} \mathrm{I} \& \mathrm{Ph} \text { II trials in } \\
\text { progress }\end{array}$ \\
\hline SAR3419 [huB4-DM4 & DM4 & huB4/CD19 & $\mathrm{NHL}^{(\mathrm{b})}$ & sanofi-aventis & $\mathrm{Ph} \mathrm{I}$ \\
\hline
\end{tabular}

(a) initiated in collaboration with British Biotech.

(b) $\mathrm{AML}=$ Acute Myeloid Leukemia; SCLC = Small Cell Lung Cancer; NHL = Non-Hodgkin's Lymphoma.

(b) DM1 $=\mathrm{N}^{2 \prime}$-deacetyl- $\mathrm{N}^{2 \prime}$-[3-mercapto-1-oxopropyl]-maytansine.

(d) DM4 $=\mathrm{N}^{2 \prime}$-deacetyl- $\mathrm{N}^{2 \prime}$-[4-mercapto-4-methyl-1-oxopentyl]-maytansine.

and the nature of the linker itself - i.e., cleavable versus non-cleavable (under usual conditions of temperature, $\mathrm{pH}$, and chemical environment in biological systems). For the latter point, the considerations are that an active cytotoxic agent must be released in target cells to kill them, yet the linker must have sufficient stability in vivo in plasma to allow distribution of the maytansinoid to the tumor as the tumor-targeting antibody distributes to, and is retained by, the antigen-bearing tumor.

One approach adopted by ImmunoGen, Inc., for its AMCs is to utilize linkers that form stable covalent bonds upon reaction with lysine amino groups of antibodies. The attachment sites can be identified readily by peptide mapping techniques utilizing mass spectroscopy [4], one can optimize the number of linkers attached over a wide range, the reactions can be readily controlled once an optimal number of linkers is determined, and such reactions (usually with $\mathrm{N}$ hydroxysuccinimide esters) leave untouched the interchain disulfide bonds of the antibody that may be important for antibody stability.

The optimal number of maytansinoid drugs linked per antibody may depend on the antibody/antigentarget pair, and can be readily determined by empirical observation. In our experience with several antibodies, in studies with conjugates with varying amounts of maytansinoids linked per antibody, the in vivo activity in xenograft models is greatly diminished when fewer than three molecules of the cytotoxic payload are linked per antibody molecule. The upper range of drugs linked per antibody may depend on the solubility characteristics of the AMCs.

The nature of the linkers confers important properties to the AMCs. One can tailor the design of the disulfide bond stability of the linker with the goal of maximizing the therapeutic window of the conjugates. By varying the degree of hindrance - done by successively replacing the four hydrogen atoms on the carbon atoms on either side of the linker disulfide bond with bulkier methyl groups - one can vary the susceptibility of the disulfide bond to reductive cleavage, such cleavage being hindered by the bulkier methyl groups. As one increases the degree of hindrance, one decreases the rate of reductive cleavage of the disulfide by up to 22,000-fold as measured biochemically, and in vivo, the terminal half-life of clearance of intact AMC from blood plasma in mice increases from about 0.7 days until, at about 7-8 days, it is similar to the clearance of "naked" human $\operatorname{IgG}_{1}$.

However, the half-life of the linker is not the only parameter to examine. We have found that the location of the methyl groups matters also. For many antibodies, we have found that AMCs with the methyl group hindrance on the payload side of the disulfide link have clearly superior antitumor activity in vivo to those with the hindrance located on the antibody side of the disulfide link, despite the fact that the AMCs have identical chemical stability of the disulfide link, and identical clearance properties from blood plasma.

Another linker option is a non-cleavable link, utilizing the thiol of the maytansinoid to form a thioether link with, for example, a maleimido group such as present in the crosslinking agent, SMCC. As expected, AMCs with non-cleavable linkers show slow clearance and a long half-life in vivo. The SMCC conjugates and disulfide conjugates of the huC242 antibody have similar in vitro cytotoxicity, explained by the finding that initial release of maytansinoid from the antibody in either conjugate is by degradation of the antibody moiety of the AMC in the lysosome of the cell [5]. However, in 
an example of the trade-offs one must make, the noncleavable SMCC linker results in a huC242 antibody conjugate that is far less active at killing colon tumor xenografts in vivo than a disulfide-linked conjugate despite the similar in vitro activity and the fact that the SMCC conjugate has a much longer half-life. Further in vitro and in vivo studies suggest that the maytansinoid moieties ultimately released from disulfide-linked conjugates, in contrast to that released from a thioetherlinked conjugate, allow bystander killing of neighboring cells $[5,6]$. We conclude that for tumors targeted by the huC 242 antibody, the bystander-killing mechanisms are important for delivery of maytansinoid to all cells in a tumor in order to achieve complete eradication of the tumor. The bystander-killing mechanism may overcome various barriers to the even distribution of AMC to all cells within a tumor.

However, when it comes to linkers, "one size does not fit all". When Genentech studied trastuzumab linked to maytansinoids, their evaluation led to their selection of the AMC comprising DM1 and the SMCC linker to advance into clinical trials [7]. Thus, one must evaluate the best design of linker for each antibody. Indeed, the three AMCs that have advanced to Phase II clinical evaluation [Table 1] are conjugates with three different designs of maytansinoid-linker, each tailored to suit the particular biological characteristics of the target antigen and the characteristics of the tumor cells that express the target.

Clinical Evaluation of AMCs: Currently five AMC compounds are in clinical testing using ImmunoGen's maytansinoid technology (Table 1), with several more AMCs in preclinical development at several of ImmunoGen's corporate partners. Phase I studies have shown AMCs are well tolerated with remarkably little hematologic toxicity despite the high concentration of conjugated antibody maintained in the blood plasma.

One of the compounds in the clinic, trastuzumabMCC-DM1, is a conjugate of the maytansinoid DM1 with an antibody, trastuzumab, that is already an approved product as a "naked" antibody. This AMC compound is being evaluated in patients with HER2expressing metastatic breast cancer that has progressed on or within 60 days of treatment with a chemotherapy regimen that includes Herceptin ${ }^{\circledR}$ (trastuzumab). Thus, trastuzumab-MCC-DM1 is being evaluated in patients whose cancer is known to not be responsive to its component antibody, trastuzumab. The initial clinical data reported is very encouraging [7], and includes objective evidence of anticancer activity in multiple Herceptin ${ }^{\circledR}$-nonresponsive patients.
Conclusion: The ability to tailor the design of each AMC compound to maximize therapeutic window - further expanding the potential for the field has improved markedly in recent years through additions made to ImmunoGen's portfolio of maytansinoid agents and linkers. Some thirty years after the discovery of monoclonal antibodies, this generation of highly potent compounds has great potential to be effective treatments for many cancers.

\section{References}

[1] W. Blättler and R. Chari: Drugs to enhance the therapeutic potency of anti-cancer antibodies: antibody-drug conjugates as tumor-activated prodrugs, in: Anticancer Agents - Frontiers in Cancer Chemotherapy, Iwao Ojima, Gregory D. Vite and Karl-Heinz Altmann, eds, American Chemical Society, 2001, pp. 317-338.

[2] C. Liu, M. Tadayoni, L. Bourret, K. Mattocks, S. Derr, W. Widdison, N. Kedersha, P. Ariniello, V. Goldmacher, J. Lambert, W. Blättler and R. Chari, Eradication of large colon tumor xenografts by targeted delivery of maytansinoids, Proc Natl Acad Sci USA 93 (1996), 8618-8623.

[3] P. Tassone, A. Gozzini, V. Goldmacher, M. Shammas, K. Whiteman, D. Carrasco, C. Li, C. Allam, S. Venuta, K. Anderson and $\mathrm{N}$. Munshi, In vitro and in vivo activity of the maytansinoid immunoconjugate hun901- $\mathrm{N}^{2}$-deacetyl- $\mathrm{N}^{2}$-(3-mercapto1-oxopropyl)-maytansine against CD56+ multiple myeloma cells, Cancer Res 64 (2004), 4629-4636.

[4] L. Wang, G. Amphlett, W. Blättler, J. Lambert and W. Zhang, Structural characterization of the maytansinoid-monoclonal antibody immunoconjugate, huN901-DM1, by mass spectrometry, Protein Sci 14 (2005), 2436-2446.

[5] H. Erickson, P. Park, W. Widdison, Y. Kovtun, L. Garrett, K. Hoffman, R. Lutz, V. Goldmacher and W. Blättler, Antibodymaytansinoid conjugates are activated in targeted cancer cells by lysosomal degradation and linker-dependent intracellular processing, Cancer Res 66 (2006), 4426-4433.

[6] Y. Kovtun, C. Audette, Y. Ye, H. Xie, M. Ruberti, S. Phinney, B. Leece, T. Chittenden, W. Blättler and V. Goldmacher, Antibody-drug conjugates designed to eradicate tumors with homogeneous and heterogeneous expression of the target antigen, Cancer Res 66 (2006), 3214-3221.

[7] M. Beeram, I. Krop, S. Modi, A. Tolcher, N. Rabbee, S. Girish, J. Tibbitts, S. Holden, S. Lutzker and H. Burris, A phase I study of trastuzumab-MCC-DM1 (T-DM1), a first-in-class HER2 antibody-drug conjugate (ADC), in patients (pts) with HER2 + metastatic breast cancer (BC), J Clin. Oncol, 25, Proceedings of ASCO, Abstract \#1042, 2007.

[14.50-15.10]

Blocking neuropilin-1function phage antibodies has an additive effect with anti-VEGF to inhibit tumor growth

Yan $\mathrm{Wu}$

Department of Antibody Engineering, Genentech Inc, South San Francisco, CA, USA 
Neuropilin-1 (NRP1) was first identified as a receptor for class 3 semaphorines and function in a variety of neural wiring processes, it has also been shown to be a receptor for specific isoforms of VEGF. To investigate therapeutic potential of blocking NRP1 in tumor angiogenesis, we generated two cross species phage antibodies that bind to the sema- and VEGF- binding domains of NRP1, respectively. Three aspects of the project will be presented: 1) Design a synthetic antibody phage library; generate, characterize and optimize of anti-NRP1 phage antibodies. 2) Anti-NRP1 phage antibodies block neovascularization, and inhibit vascular remodeling in the developing retina. AntiNRP1 and anti-VEGF combination has additive effect in several human xenograft tumor models. 3) Crystal structure of antibody and antigen complex will be discussed.

\section{[15.10-15.25]}

\author{
AFRA-DFM a new completely human antibody \\ fragment for ovarian tumor targeting of \\ radionuclides \\ M. Figini ${ }^{\mathrm{a}}$, F. Martin ${ }^{\mathrm{b}}$, A. Zacchetti ${ }^{\mathrm{a}}$, E. Luison ${ }^{\mathrm{a}}$, A. \\ Coliva $^{\mathrm{a}}$, E. Bombardieri ${ }^{\mathrm{a}}$, M. Mortarino ${ }^{\mathrm{a}}$, M. \\ Cattozzo $^{\mathrm{b}}$, G. Maurizi ${ }^{\mathrm{b}}$, V. Di Cioccio ${ }^{\mathrm{b}}, \mathrm{M}$. \\ Allegretti $^{\mathrm{b}}$ and S. Canevari ${ }^{\mathrm{a}}$ \\ ${ }^{a}$ Unit of Molecular Therapies, Fondazione IRCCS \\ Istituto Nazionale dei Tumori, Milan, Italy \\ Tel.: +3902 23902567; E-mail: \\ silvana.canevari@istitutotumori.mi.it \\ ${ }^{\mathrm{b}}$ Dompé Pha.r.ma, L'Aquila, Italy
}

Introduction: Monoclonal antibodies therapies have recently positively impacted and become standard elements in the treatment of hematological and certain solid malignancies [1]. Such advances have shown only a marginal value in the epithelial ovarian carcinoma (EOC) treatment [2]. Our group developed during the last years several murine and chimeric antibodies against a specific EOC marker, named folate receptor (FR) $[3,4]$ and promising results were obtained in a phase II trial with a radioimmunotherapic (RIT) approach [5]. RIT has two major advantages: i) radionuclides can irradiate not only specifically targeted tumor cells but also surrounding area including tumor cells not expressing the antigen of interest; ii) the mechanism of action is not dependent from drug resistance. Thus, taking in consideration antibody origin, size, half life and affinity and the peculiar EOC tumor localization we propose the use of human antibody fragments (Fab) in a dimer format for an EOC locoregional RIT approach. Indeed, the Fab small dimension is likely to favor tumor penetration and shortened half-life are expected; its human origin should eliminate immunogenic reactions; moreover, this type of molecule does not require any glycosylation to be functional and accordingly is compatible with micro-organism production and the associated advantages. Shifting from the Fab to the dimer format gives further advantages, such as bivalency, which increases its overall binding strength, doubles its molecular weight with respect to the corresponding Fab fragment, which in turn decreases excretion in urine protecting kidneys and consequently prolongs its in vivo half-life. Here are shortly described the adopted strategy for its generation and its in vitro and in vivo preclinical validation.

Strategy adopted for generation and production of AFRA-DFM: Initially we considered as candidate for in vivo clinical use the already described anti-FR human Fab fragment $\mathrm{C} 4$ [6] and in order to stabilize the bivalent format in vivo bismaleimide ethane [7], a non hydrolysable linker, was used to convert the Fab' fragment into a Di Fab Maleimide (DFM). The C4 chemical dimerisation reaction gives a yield of $<5 \%$, of which only about $30 \%$ is the correct dimer. The unexpected very low yield of C4-DFM formation hampered any further clinical development of $\mathrm{C} 4$. Thus a new antiFR human Fab fragment was produced adopting the strategy depicted in Fig. 1. An antibody library, potentially biased toward an-antitumor response, was generated starting from peripheral blood lymphocytes of EOC patients, who achieved a long term complete response following surgical and chemotherapic first-line treatment [8]. The human heavy chain previously selected [6] was paired with the kappa light chain antibody library and used to guide selection on EOC FRexpressing cells. The selected Fab fragment, named AFRA, could be produced in Escherichia coli at high yield and the overall $\%$ of chemical dimer obtained at the end of the reaction is $>10$-fold higher than that obtained with C4. AFRA-DFM can be obtained at $>90 \%$ purity of chemical $\mathrm{F}(\mathrm{ab})_{2}$.

AFRA-DFM in vitro characterization: AFRA-DFM specifically bound to FR expressing tumor cells and competed with the original anti-FR mouse Mab used to guide its selection. The affinity of the monomer was in the nanomolar range $(44 \mathrm{nM})$ and the overall affinity, in the dimer format, was improved. In an in vivo context, by immunohistochemistry it was able to recognize in situ the FR expressed on EOC samples. AFRADFM production and purification was scaled-up in clin- 
Table 1

AFRA-DFM in vivo preclinical validation

\begin{tabular}{|c|c|c|c|c|}
\hline \multirow[t]{2}{*}{ Preclinical in vivo models } & \multicolumn{2}{|r|}{ Localization\# } & \multicolumn{2}{|c|}{ Efficacy* } \\
\hline & $\mathrm{N}^{\circ} \exp$ & $\begin{array}{c}\% \text { ID/g * solid tumor } \\
(\text { mean } \pm \mathrm{SE})\end{array}$ & $\mathrm{N}^{\circ} \exp$ & $\begin{array}{l}\% \text { reduction tumor } \\
\text { take }(\text { mean } \pm \mathrm{SE})\end{array}$ \\
\hline \multicolumn{5}{|l|}{ Subcutaneous xenotransplants of: } \\
\hline A431-FR cells & 2 & $6.17 \pm 0.36$ & 5 & $52.40 \pm 2.87$ \\
\hline A431-Mock cells & 2 & $2.84 \pm 0.60$ & 3 & $10.69 \pm 0.62$ \\
\hline & $\mathrm{N}^{\circ} \exp$ & $\begin{array}{l}\% \mathrm{ID} / \mathrm{g} \text { tumor cells in } \\
\text { ascite }(\text { mean } \pm \mathrm{SE})\end{array}$ & $\begin{array}{c}\mathrm{N}^{\circ} \exp \\
\text { increase overall survival }\end{array}$ & mean $\%$ \\
\hline \multicolumn{5}{|l|}{ Intraperitoneal xenotransplants of: } \\
\hline IGROV-1 cells & 3 & $25.10 \pm 1.33$ & 2 & 100 \\
\hline OVCAR-3 cells & 1 & 13.2 & 2 & 89 \\
\hline
\end{tabular}

§Athymic mice were xenografted subcutaneously with $2.5 \times 10^{6}$ A431-FR or A431-Mock, for specificity evaluation, [ref Coliva], and intraperitoneally with $8-10 \times 10^{6}$ IGROV1 or OVCAR-3 cells. The mean number of animals for each experiment was 3-4 for localization and 6-8 for efficacy.

\#For localization experiments ${ }^{131}$ I-AFRA-DFM was injected at radiotracer dose (30-40 microCi/mouse): in s.c models by intravenous administration when tumors reached a mean volume of $0.2-0.3 \mathrm{~cm}^{3}$; in ip models by ip administration when ascite formation becomes evident (14-20 days after tumour cell injection). Results are expressed as the percentage of the injected dose per gram of tissue or pellet of cells $(\% \mathrm{ID} / \mathrm{g})$ measured 3 hours following radiotracer injection and are compensated for radioactivity decay.

*For efficacy evaluation ${ }^{131}$ I-AFRA-DFM was injected at therapeutic dose $(1 \mathrm{mCi} / \mathrm{mouse})$ : in s.c models by intravenous administration1 week after tumor injection when tumors reached a mean volume of $0.04-0.05 \mathrm{~cm}^{3}$; in ip models by ip administration 2 or 4 days after tumor injection. Results are expressed: for sc models as the percentage of tumor reduction relative to untreated animals at day 11-17 after antibody administration; for ip models as percentage of increase in overall survival relative to untreated animals (median survival time in controls: IGROV1: 18 days; OVCAR-3: 19 days).

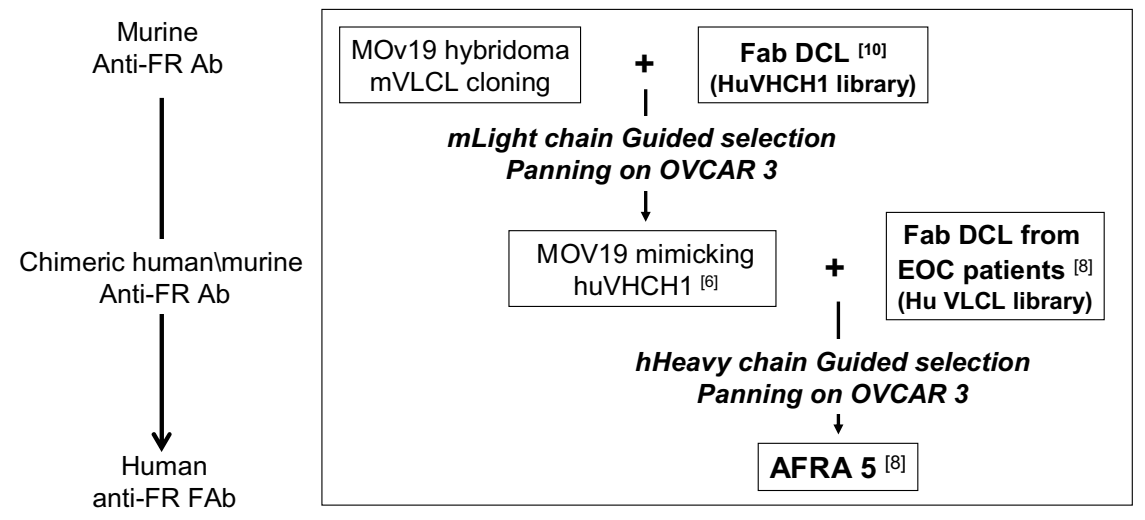

Fig. 1. Scheme of the strategy adopted to select the new human anti-FR Fab, named AFRA.

ical grade conditions (Martin et al manuscript preparation) and radiolabeling with ${ }^{131}$ I using the Chizzonite method enabled to preserve the integrity and functionally of the molecule; in fact, the final radiolabeled product, at a specific activity of $4-5 \mathrm{mCi} / \mathrm{mg}$, showed a mean immunoreactivity of at least $65 \%$. All these data argued for the AFRA-DFM potential suitability for RIT.

AFRA-DFM in vivo preclinical validation: In two different preclinical in vivo models AFRA-DFM recognized only tumors expressing the antigen of interest and ${ }^{131}$ I-AFRA-DFM when injected as radiotracer resulted in a specifically accumulation in FR expressing tumor cells, particularly evident on ascitic cells (Table 1). When administered at therapeutic doses ${ }^{131} \mathrm{I}-$ AFRA-DFM significantly reduced tumor growth or increased overall survival of treated animals in subcutaneous or intraperitoneal models respectively (Table 1) (Figini et al manuscript preparation).

Concluding remarks: Since ovarian cancer has a high propensity to stay confined to the peritoneal cavity until very late in the course of the disease, radiolabeled antibodies have been administered intraperitoneally in many trials. The overall data emerging from these trials (reviewed in [9]) support the concept that RIT can have a role in the treatment of small lesions of ovarian cancer 
and minimal residual disease. In this framework, the here presented data, upon further optimization of doses and schedules of treatment, point to the promise of intraperitoneal RIT with AFRA-DFM in the management of ovarian cancer.

\section{References}

[1] P. Carter, Potent antibody therapeutics by design, Nat Rev Immunol 6(5) (May 2006), 343-357.

[2] R.H. Verheijen, L.F. Massuger, B.B. Benigno, A.A. Epenetos, A. Lopes, J.T. Soper, J. Markowska, R. Vyzula, T. Jobling, G. Stamp, G. Spiegel, D. Thurston, T. Falke, H. Lambert and M.V. Seiden, Phase III trial of intraperitoneal therapy with yttrium-90-labeled HMFG1 murine monoclonal antibody in patients with epithelial ovarian cancer after a surgically defined complete remission, J Clin Oncol 1;24(4) (Feb. 2006), 571578.

[3] S. Miotti, S. Aguanno, S. Canevari, A. Diotti, R. Orlandi, S. Sonnino and M.I. Colnaghi, Biochemical analysis of human ovarian cancer-associated antigens defined by murine monoclonal antibodies, Cancer Res 45(2) (Feb. 1985), 826-832.

[4] L.R. Coney, A. Tomassetti, L. Carayannopoulos, V. Frasca, B.A. Kamen, M.I. Colnaghi and V.R. Zurawski Jr., Cloning of a tumor-associated antigen: MOv18 and MOv19 antibodies recognize a folate-binding protein, Cancer Res 15;51(22) (Nov. 1991), 6125-6132.

[5] F. Crippa, G. Bolis, E. Seregni, N. Gavoni, E. Bombardieri, G. Scarfone, C. Ferrari and G.L. Buraggi, Single dose intraperitoneal radioimmunotherapy with the murine monoclonal antibody 131I-MOv18: clinical results in patients with minimal residual disease of ovarian cancer, Eur J Cancer 31A (1995), 686-690.

[6] M. Figini, L. Obici, D. Mezzanzanica, A. Griffiths, M.I. Colnaghi, G. Winter and S. Canevari, Selection of human Fab fragment from naive library directed against a cell surface tumour antigen overexpressed in ovarian, Cancer Res 58 (1998), 991-996.

[7] M.A. Stalteri and S.J. Mather, A cross-linked monoclonal antibody fragment for improved tumor targeting, Bioconjug Chem 6(2) (Mar-Apr, 1995), 179-186s

[8] M. Figini et al., Successful conversion of a murine antibody into a new human antibody and its optimization for ovarian cancer radioimmunotherapy targeted to folate receptor, submitted.

[9] Angela Coliva, Ettore Seregni, Mariangela Figini, Alberto Zacchetti. Rosanna Fontanelli, Francesco Raspagliesi, Silvana Canevari and Emilio Bombardieri, Nuclear medicine in the treatment of ovarian cancer in: Advances in Nuclear Oncology: Diagnosis and Therapy Edited by Emilio Bombardieri

[10] M. Figini, J.D. Marks, G. Winter and A. Griffiths, In vitro assembly of repertoires of antibody chains on the surface of phage by renaturation, J Mol Biol 239 (1994), 68-78.

\section{[15.25-15.40]}

\section{Induction of NeuGc-containing gangliosides} related idiotypic networks in NSCLC patients immunized with an anti-idiotype antibody Ana María Hernández, Darién Toledo Santamaría, Rosa María Díaz, Amparo Macías, Tania Griñán, Teresa Rondón, Ana María Vázquez and Rolando Pérez

\section{Centre of Molecular Immunology, Havana, Cuba}

$1 \mathrm{E} 10 \mathrm{MAb}$ is an anti-idiotype murine monoclonal antibody (Ab2 MAb) specific for an Ab1 MAb which reacts with NeuGc-containing gangliosides, sulfatides and with antigens expressed in some human tumors. Previous results reported by our group proved that the immunization of melanoma and breast cancer patients with this antibody precipitated in aluminum hydroxide, induced a specific response against 1E10 idiotype and $\mathrm{NeuGcGM}_{3}$ ganglioside. Furthermore, when patient sera were adsorbed with $1 \mathrm{E} 10 \mathrm{MAb}$, a remnant reactivity against $\mathrm{NeuGcGM}_{3}$ was still detected suggesting the presence of antibodies Id-Ag+. The present work shows new data about the immune response elicited in 20 NSCLC patients, treated with $1 \mathrm{mg}$ of aluminum hydroxide-precipitated 1E10 MAb. In the hyperimmune sera from 18 out of 20 patients, a strong specific antibody response of both IgM and IgG isotypes against NeuGcGM3 ganglioside was observed. Patient immune sera were able to induce a complementindependent cell death, in the NeuGcGM3-containing X63 myeloma cell line. Anti-NeuGcGM3 IgG and IgM antibody responses correlated with survival of vaccinated patients. A significant immunoreactivity to NeuGcGM3 was still detected after the complete abrogation of the reactivity against the $1 \mathrm{E} 10 \mathrm{MAb}$ by the adsorption of patient sera with this antibody. Both fractions, $\mathrm{Id}+\mathrm{Ag}+$ and $\mathrm{Id}-\mathrm{Ag}+$, were separated by affinity chromatography and further characterized. While both $\mathrm{IgG}$ and $\mathrm{IgM}$ isotypes were found in the Id-Ag+ fraction, IgM antibodies to NeuGcGM3 were not detected in the "true" Ab3 response ( $\mathrm{Id}+\mathrm{Ag}+$ fraction). AntiNeuGcGM3 IgG antibody titers were similar or even higher in the Id-Ag+ fraction as compared with the "true" Ab3 response (Id+Ag + fraction), suggesting an amplification mechanism of the immune response to the anti-idiotype antibody used as immunogen. These results could be explained assuming the induction of an idiotypic cascade in vaccinated patients. We hypothesise that $\mathrm{Id}-\mathrm{Ag}+$ antibodies reflect the Ab5 response. The clinical relevance of such idiotypic cascade deserves further investigation. 